\title{
Brain tumor surgery: supplemental intra-operative imaging techniques and future challenges
}

\author{
Telmo Augusto Barba Belsuzarri, Raphael Martinelli Anson Sangenis, João Flavio Mattos Araujo \\ Department of Neurosurgery, Pontifical Catholic University of Campinas, Campinas, São Paulo 13060-803, Brazil.
}

Correspondence to: Dr. Telmo Augusto Barba Belsuzarri, Department of Neurosurgery, Pontifical Catholic University of Campinas, Campinas, São Paulo 13060-803, Brazil. E-mail: telmobelsuzarri@hotmail.com

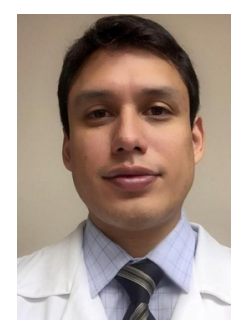

Telmo Augusto Barba Belsuzarri, MD, is a Neurosurgery Resident at the Pontifical Catholic University of Campinas (PUC-CAMPINAS). Graduated in Neurocritical Care, with thesis in Magnesium Sulphate in Neurointensive care, Dr. Belsuzarri has published papers in several areas and has special interest in Neurooncology/Cranial Base, Vascular Neurosurgery and Functional Neurosurgery.

\section{A B S T R A C T}

Modern brain tumor surgery stands in the pillar of maximum safe resection. Tumor borders are always challenging, especially infiltration zones in malignant brain tumors. Novel technologies are designed for a better delineation and to increase the extent of resection (EOR) in brain tumor surgery, such as: cortical and sub-cortical mapping strategies with somatosensory-evoked potentials, awake stimulation mapping and cortical/sub-cortical stimulation for motor pathways, important for resection in eloquent areas; intra-operative imaging as functional and intra-operative magnetic resonance imaging, diffusion tensor imaging and intraoperative ultrasound are important for the tumor borders and to achieve the gross total resection; neurochemical navigation methods as 5-aminolevulinic and sodium fluorescein are important for the non-contrast-enhanced tumor border; future methods can be achieved with augmented reality surgery, new intra-operative chemical markers, and visualization methods. Nevertheless all these techniques seem to be promising, the real challenge in the future will be held in how to apply them and how they really affect the prognosis of the patients. Also, new concepts in tumor genetics will provide knowledge for the tumor behavior and will guide resection. Despite all limitations, the increasing importance of safe EOR shows the possible benefits of the novel technologies and surgical advances in brain tumor surgery, taking it to a new step of the neuronavigation era.

Key words: Brain tumor; fluorescein; intra-operative; neuronavigation; novel; technology

\section{INTRODUCTION}

Neurosurgery went through several changes over the past 50 years; technology has been applied to all fields, since the introduction of microscope and the microsurgical technique by Yasargil, until endoscopes, minimally invasive spine surgery and functional neurosurgery with deep brain stimulation implants. As we see, the neurosurgery has two important arms in this modern era: the equipment and the surgical expertise.

\begin{tabular}{|l|l|}
\hline \multicolumn{3}{|c|}{ Access this article online } \\
\hline Quick Response Code: & Website: \\
\hline & www.jcmtjournal.com \\
\cline { 2 - 2 } & \\
\hline
\end{tabular}

New imaging technologies are applied to other two different manners, pre-surgical moment and intra-operative imaging. ${ }^{[1]}$

Modern neurosurgery lives a paradigm of concepts. Although there are insufficient proves of the real benefits and impacts of the aggressive image-guided neurosurgery, ${ }^{[2]}$ evidences show the importance of gross total resection (GTR) in the quality of treatment and the effectiveness

This is an open access article distributed under the terms of the Creative Commons Attribution-NonCommercial-ShareAlike 3.0 License, which allows others to remix, tweak, and build upon the work non-commercially, as long as the author is credited and the new creations are licensed under the identical terms.

For reprints contact: service@oaepublish.com

How to cite this article: Belsuzarri TAB, Sangenis RMA, Araujo JFM. Brain tumor surgery: supplemental intra-operative imaging techniques and future challenges. J Cancer Metastasis Treat 2016;2:70-9.

Received: 29-09-2015; Accepted: 30-11-2015. 
increase of adjuvant therapy. ${ }^{[3-5]}$

Neuroimaging has been playing an important role in neurosurgery in the last century and technology has come to provide details in neuroanatomy, neurological function, metabolic and metabolism, which augments the ability to increase the extent of resection (EOR) while simultaneously, minimizes the risk of damage in eloquent brain. ${ }^{[1]}$

Increasing evidences show the importance of GTR for glioblastoma multiforme (GBM's) and adjuvant radiochemotherapy and demonstrate a 2-8 months survival benefit in patients with GTR compared to sub-total resection. ${ }^{[3-5]}$ Nevertheless, after a first impact, the focus has changed from just increasing the EOR, to increase the quality of life and safe resection; the tumor relationship critical anatomic structures and eloquent areas have become the center of the discussion.

Nowadays, molecular genetics came to open further discussions about tumor behavior, such as isocitrate dehydrogenase mutations (IDH) $1 / 2,1 \mathrm{p} 19 \mathrm{q}$ codeletion, PTEN deletions, MGMT mutation, telomerase reverse transciptase (TERTp) mutation, EGFR and TP53. ${ }^{[6-8]}$ The IDH 1 and 2, were first described in GBM's, nevertheless further research showed that it was more expressive in grade II-III diffuse gliomas (about 70\%). IDH 1/2 mutations are important biomarkers for diffuse gliomas, because they behave less aggressively and a better prognosis, than other IDH mutations (IDH wild-types), with a positive predictive value (PPV) for better progression-free survival and overall survival. ${ }^{[6]}$ The $1 \mathrm{p} / 19 \mathrm{q}$ codeletion is found in almost $70 \%$ of histologically oligodendrogliomas, thus have an indolent progression and might be the molecular defi nition of oligodendroglial lineage. Also, these low-grade gliomas (LGG) tend to respond well for chemotherapy, thus have better prognosis. The MGMT is an enzyme, which repairs the DNA and interferes in temozolamide effect. Mutations in the MGMT have been correlated with improved prognosis and improved survival rate. ${ }^{[6,7]}$ On the other hand the ATRX/TP53 mutations might be the objective genetical markers of the artrocytic lineage. ${ }^{[6,7]}$

In GBM's the most common aberrations are found in the chromosomes 7 and 10, where the PTEN and EGFR are located. Aberrations on the PTEN and EGFR amplifications are found in $80 \%$ and $30-40 \%$, respectively, both of them strongly related to poor prognosis and aggressive progression, which reinforces the idea that these tumors with PTEN and/or EGFR amplifications are IDH-wild type tumors. ${ }^{[6,7]}$ Finally, studies points for the association of promoter region of the TERTp mutation and poor prognosis. ${ }^{[8]}$ A recent research was published comparing the TERTp mutation, $1 \mathrm{p} 19 \mathrm{q}$ codeletion and IDH mutation in Grade II-III and GBM's with interesting findings. ${ }^{[8]}$ TERTp mutation only, was found in 347 patients with GBM's, compared to the TERT and IDH group with 11 patients, IDH mutation only group with 32 patients and triple negative group with 80 patients. This data shows that almost $75 \%$ of the patients with GBM have only TERT mutation and have a correlation with aggressive behavior type of gliomas. Also, patients with Grade II-III with TERT mutation only (59 patients), had an aggressive course and were associated with poor survival, which suggest the need of early adjuvant therapies and special follow-up. Also only IDH mutation, was associated with lowest age of diagnosis (37 years) and the highest rates were found in the only TERT mutation group (59 years), between all the gliomas. This study opens for further research between the association of TERTp and other previously discussed mutations. ${ }^{[8]}$

The genetic studies and imaging findings have become allies in the understanding of tumor behavior; nevertheless have also pointed questions on the efficiency of the surgical techniques to improve patient prognosis and the natural history of these tumors.

\section{METHODS}

A literature search of the Ovid Medline and PubMed databases for the period January 1980 to September 2015 was conducted using the following key words: brain tumor, borders, technology, neuronavigation, intra-operative, fluorescein, novel. Main novel technologies were selected by their relevance and were analyzed by categories.

\section{RESULTS}

Neurosurgery has rapidly changed in the past years due to new technologies and new different possible surgical approaches. These changes have modified neurosurgical concepts, from an aggressive vision to a safe EOR with good function. Since the beginning of the microsurgical era, the surgical planning has improved from an anatomyplanned surgery to an optimal non-visible tumor borders resection. ${ }^{[1,2]}$

Several technologies were introduced in the intra-operative field such as functional monitoring with cortical and subcortical mapping, imaging technologies as neuronavigation, intra-operative magnetic resonance imaging (iMRI), intraoperative ultrassound (iUS), chemical biomarkers as 5-aminolevulinic acid (5-ALA), and sodium fluorescence. ${ }^{[2]}$

Some of these advances were possible not only for the technology, but also due to anesthetic advances and better neurofunctional knowledge..$^{[9,10]}$

Nevertheless, even with the standard care in neurosurgery, the 2-year survival rate in GBM's still is about $38.4 \%$ and the 5-year rate is below 5\%. ${ }^{[11-13]}$ Also, past reports have shown that even with hemispherectomies, patients could not be cured. ${ }^{[14,15]}$ Even though, the surgical technology has improved the past years, there are no consistent evidences 
of improving survival rate. ${ }^{[2]}$

In this point we have two arms, the technologies to improve resection and to increase the knowledge of tumor nature. By now it is clear that just improving resection won't provide the best result, but better understanding of the different diseases and tumor natures, will provide direction for optimal resections and better outcomes.

\section{Awake craniotomy}

Anesthetic advances permitted safer awake craniotomies to obtain brain mapping and better neurosurgical borders. However, it has a series of challenges to be analyzed before such as integration of different types of knowledge, imaging, multidisciplinary team, cooperation from several clinics sectors, application of protocols, application of specific technical adjustments, and a multidisciplinary approach. The integration of the pre-operative functional MRI (fMRI) and neuropsychological tests are the key for a good planning and patient selection. Not all tumor patients should undergo awake craniotomy, but patients with lesions close relationship with eloquent areas, in special for motor and speech. ${ }^{[16,17]}$ Talacchi et al. stated that intra-operative complication can vary from anesthetic (inadequate or excessive sedation, pain, nausea, vomiting); respiratory (oxygen saturation $<90 \%$, increased $\mathrm{CO}_{2}$, hypoventilation $<8$ breaths/min, airway obstruction); hemodynamic (hyperor hypotension, tachy- or bradycardia); and neurological (convulsions, brain swelling, new neurological deficit). From these complications, hyper- and hypotension are the most frequent in awake surgery (11\% and 56\%, respectively)..$^{[16,17]}$

The main purpose of awake surgery is the monitoring of speech and motor pathways. This way, the physical preoperative imaging/clinical examinations and intra-operative positive tests are important. Patients with aphasias and language disturbance seen at the physical examination, have higher risk of post-operative neurological deterioration. Intra-operative positive tests for stimulation in motor areas have also higher risk of motor deterioration, probably due to the proximity of the tumor lesion to the cortical tracts. ${ }^{[16]}$

Shinoura et al. studied motor worsening after 102 motor areas glioma surgery; they have encountered motor worsening immediately after surgery and after 1 month were related to awake surgery failure and intra-operative complications. The main causes of failure of awake surgery are severe somnolence, epilepsy, air embolism, no wake up and motor neglect. ${ }^{[18]}$

In order to analyze the hemisphere dominance, the Edinburgh, Wada or fMRI (with verb generation tasks) can be done. Also, multiple tests are applied to the language task with visual object naming tests such as the Boston naming test, Snodgrass and Vanderwart Test, DO80, and Aachner Aphasia Test. They are done to map the dominant hemisphere and localization of speech areas. The pre-motor areas of the face are always tested to identify possible motor causes of the aphasia. Even with all protocols, intra-operative positive sites errors can range from $4.6 \%$ to $22 \% .{ }^{[16]}$ The counting test is used to document a speech arrest during electrocortical stimulation and also auditory naming, verb generation and reading are commonly used tests. Additional tests can be applied such as calculation, visuospatial functions, working memory, visual pathways, eye movements, and writing. ${ }^{[16,17]}$

One important point is that function can be found at the edge of high-grade gliomas and also within the tumor in low-grades, so it has to be analyzed for a safe EOR. ${ }^{[19,20]}$

Awake surgery has been used for some time, but new tests and anesthetic evolution have permitted a better understanding of functional areas and also the mapping of complex brain areas.

\section{Cortical and sub-cortical mapping}

During the past years, the increase importance of the EOR and the relationship with increased overall survivalhas made the neurosurgeons push to the limits of the glioma surgeries, even in eloquent areas. Nevertheless, without intra-operative monitoring, morbidity increasing became fact. The objective of increasing overall survival with good functional status made the neuronavigation era a reality. ${ }^{[1,21]}$

As imaging has increased its accuracy over the past years, neuroanatomy studies have shown a better knowledge of the sub-cortical tracts and the new mapping technologies have shown the real cortical and functional mapping, which most of the times can be changed by the lesion..$^{[9,22]}$

Intra-operative monitoring has been studied by several different methods, using somatosensory-evoked potentials (SSEP), awake stimulation, and cortical/sub-cortical direct motor stimulation. SSEP uses sub-dural electrodes to evoke potentials of gyri and to localize the central core (pre-central and post-central gyri) [Figure 1]. Awake stimulation is a

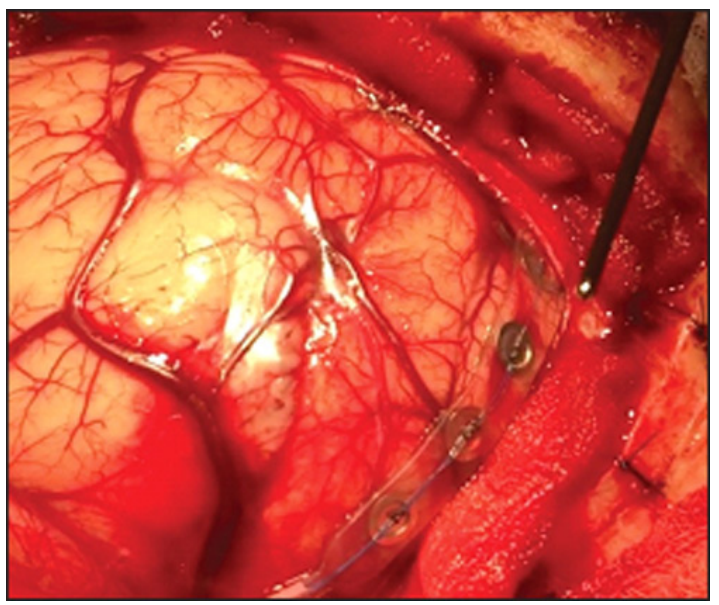

Figure 1: Direct electrical stimulation with and somatosensory-evoked potentials in motor/sensory areas 
good approach for the language function and its multiple association areas, which could be more complex and even specific for different languages/cultures. Language function is the most complex and superior level function, with multiple localizations and spread connective areas. The exact language-related cortical area is not mathematical and is individual related, especially in patients with intracranial lesions. Patients with intra-cranial tumors can have very atypical language areas related to their brain mapping conformation and compliance to the tumor. ${ }^{[22]}$ The key for brain surgery of the dominant hemisphere is the central core and language function, which has become possible. ${ }^{[22,23]}$

Brain mapping usually needs large craniotomies and longer time of surgical/anesthetic exposure, but provides multiple functional areas. ${ }^{[23,24]} \mathrm{Li}$ et al. analyzed 91 cases of brain functional area glioma surgery under direct electrical stimulation (DES) and noticed that the most commonly observed areas of counting interruption were distributed on the posterior part of the left anterior central gyrus (47.7\%), the operculum of the left inferior frontal gyrus $(24.4 \%)$, the triangular part of the left inferior frontal gyrus $(12.8 \%)$, and even the posterior part of the superior frontal gyri (4.7\%). After surgery, $46 \%$ had no post-operative dysfunction, $42.9 \%$ a brief language dysfunction, $29.7 \%$ limb movement disorder, and 1 case had a permanent disability; this shows that DES is a non-invasive accurate method. ${ }^{[22]}$ Another positive point of DES is the mapping of the sub-cortical areas because it does not have influences on brain shift or other positioning errors. ${ }^{[22]}$

Even though $\mathrm{fMRI}$ is satisfying for motor/sensitivity areas, its sensitivity is only of $59-100 \%$ and specificity of $0-97 \%$ for language areas. ${ }^{[22]}$

De Witt Hamer et al. reviewed and made a meta-analysis of surgical situations of 8,091 glioma cases and found that the rate of long-term severe neurological dysfunction sub-sequent to DES was $3.4 \%$, while the long-term severe disability rate of patients that underwent surgery without DES was $8.2 \%$. In addition, for the patients undergoing
DES, the overall resection rate and the rate of involvement of the language functional area in the resection were significantly increased. ${ }^{[23]}$

Event thought DES is a relative novel technology, is also an important research method for higher cognitive functions, such as music, calculation, memory, complex speech processes, hemispheric ignorance, perception, visual pathways and more. ${ }^{[25-29]}$

\section{Neuronavigation and intra-operative magnetic resonance imaging}

Magnetic resonance has changed the course of anatomical marks in neurosurgery; since its beginning in the early 80's, the pursuit of high field technologies for better images has become a challenge. ${ }^{[9]}$

In the neuronavigation era, planning surgery has become not only a decision on craniotomies and different approaches, but also a way to prevent and predict the final surgery with minimal injuries. This way, fMRI, positron emission tomography (PET), and diffusion tensor imaging (DTI) are important technologies. ${ }^{[1]}$

PET utilizes $\mathrm{H}_{2}{ }^{15} \mathrm{O}$ as a blood tracer to measure flow or $\left({ }^{18} \mathrm{~F}\right)$-fluorodeoxglucose uptake to measure cerebral metabolism. ${ }^{[30]}$

fMRI measures blood oxygen level dependency changes due to alterations in the ratio of the oxyhaemoglobin and deoxyhaemoglobin in the most metabolically active regions $^{[30]}$ [Figure 2].

Another image technology is the DTI, which visualizes the fiber tracts with the thermally driven motion, or diffusion of water and molecules through fibers. ${ }^{[31,32]}$

DTI and fMRI allow neurosurgeons to have functional/ eloquent areas and sub-cortical fibers related to the lesions in their pre-operative planning; therefore, functional

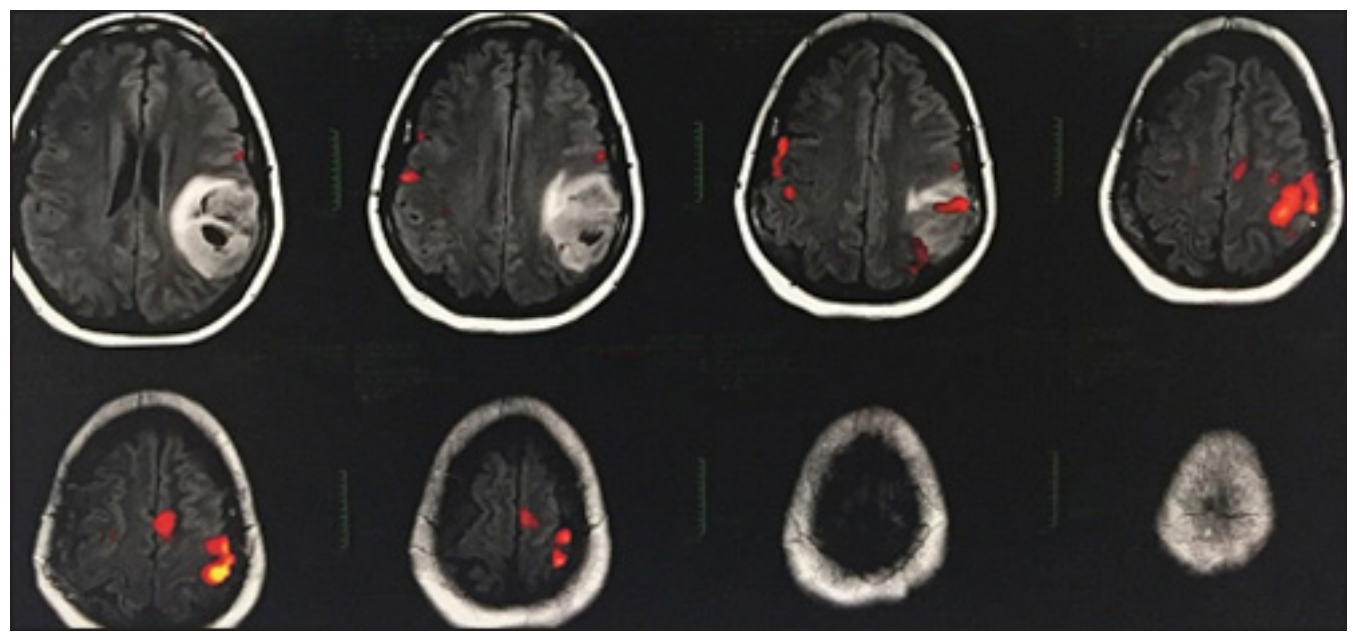

Figure 2: Functional magnetic resonance imaging and glioma: Red spots of the functional areas for the speech test near/between the tumor 


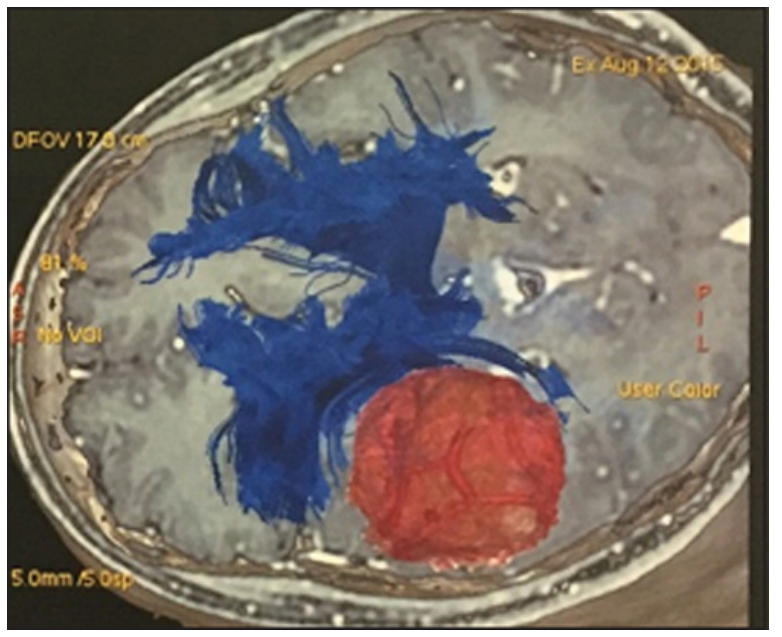

Figure 3: Tractography magnetic resonance imaging. In blue: the white fibers tracts. In red: tumor

neuronavigation has become part of the clinical decisionmaking, surgical approach, and EOR ${ }^{[33]}$ [Figure 3].

Nevertheless, the functional neuronavigation has not shown its clinical utility due to a lack of high evidence studies. Wu et al. carried out the only randomized controlled trial with an established protocol in functional neuronavigation and demonstrated a reduced post-operative motor deterioration, a higher Karnofsky Performance Scale, and an increased overall survival in study patients. ${ }^{[33]}$

One of the worst problems in neuronavigation is the brain shift; it is the change of tissue/lesion during surgery due to cerebrospinal fluid drainage, tumor resection, and brain swelling; with estimated to be around $1 \mathrm{~cm}$ after opening the dura, ${ }^{[34]}$ and more than $1 \mathrm{~cm}$ after initial resection of tumor. Therefore, the iMRI technology has come to solve this problem and also increase the EOR. The first iMRI was performed in 1994; it presented several benefits and showed that a considerable part of patients had resectable residual tumor.

In special for LGG treatment, iMRI has led to favorable results in several studies. Reports show $30-60 \%$ of return to surgery after initial resection with iMRI. ${ }^{[35-38]}$

Even though iMRI is an interesting method, nowadays there is only one randomized controlled trial comparingiMRI to conventional surgery; the trial found that iMRI was associated with higher rate of complete resection (96\% vs. 68\%) and increased progression-free survival without additional morbidity. ${ }^{[5,39]}$ Kubben et al. held a systematic review and showed just an evidence level II of iMRI being more effective than conventional neuronavigation in increasing EOR, quality of life or prolonging survival after GBM resection. ${ }^{[38,39]}$

In practical analysis, iMRI has some issues for global implementation regarding costs and time. This method requires special implementation; most of the times not only the equipment, but also revision of the local of implementation, making it a high cost technology. ${ }^{[40,41]}$ In addition, the time for image acquiring and the need of stop the surgery for it, prolong time of surgery and anesthesia. $^{[42-44]}$

Roder et al. studied retrospectively 117 patients after conventional surgery, after 5-ALA, and after iMRI they found that mean residual tumor volume after iMRI-assisted surgery $\left(0.5[0.0 \mathrm{e} 4.7] \mathrm{cm}^{3}\right)$ was significantly smaller compared to the residual tumor volume after 5-ALAguided surgery (1.9 [0.0-13.2] $\left.\mathrm{cm}^{3} ; P=0.022\right)$, which was significantly smaller than in conventional surgery (4.7 $\left.[0.0-30.6] \mathrm{cm}^{3} ; P=0.007\right)$. Total resections were significantly more common in iMRI (74\%) than in 5-ALAassisted $(46 \%, P=0.05)$ or conventional surgery $(13 \%, P$ $=0.03$ ). Also, the iMRI time of surgery was significantly higher compared to pre-iMRI period (213 vs. $354 \mathrm{~min}$ ). Improvement of the EOR using iMRI was safely achievable and post-operative morbidities were comparable between cohorts. Total resections increased 6 months progression free survival from $32 \%$ to $45 \%$. In follow-up analysis, the neuronavigation had new or worsened neurological deficits at 3 months in $18.2 \%$ of patients, compared to $45.5 \%$ of the control group. Non-neurological complications were present in both groups, $31.8 \%$ in the control group and $30.4 \%$ in the neuronavigation group. Also, the progression-free survival and survival ratewasbetter in the neuronavigation/iMRI groups vs. control groups. ${ }^{[2]}$

Despite it is a retrospective study with a short period of time and limited patients in different chronologic times, the great outcomes and promising results should open for new prospective studies. ${ }^{[42]}$ Further, the quality of iMRI images remains an issue; pre-operative MRI images are usually acquired by high-fields MRI with DTI and fMRI as a surgery plan, though intra-operative images are usually low-field MRI with worse definitions without DTI and fMRI; thereby the surgery plan for critical and eloquent areas is difficult and questionable after tumor resection and brain shift. Also, studies related to contrast dosage/ timing and the local of resection have been done. The main challenge is to differentiate tumor border from blood brain barrier brakes and surgical tissue changes, which also have contrast-enchanted borders. ${ }^{[43,44]}$ The Cochrane review point for different patients' baselines with heterogeneous lesions and the current studies do not provide quality evidences of benefits. Also, there is no standard protocol for its use and most of the time it is used in single centers. ${ }^{[2]}$

\section{Intra-operative ultrasound}

Intra-operative ultrasound is a dynamic method that can provide dynamic images with brain shift corrections and also the correlation between the tumor and normal brain, just as the tumor vascular nutrition and borders. In the past decades, the iUS increased the quality of images, from poor-quality images to three-dimensional (3D) imaging 


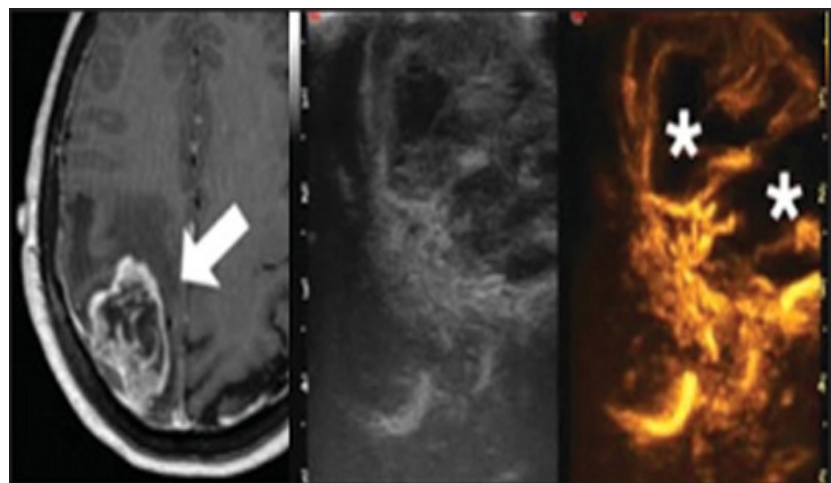

Figure 4: Contrast-enhanced magnetic resonance imaging and intra operative ultrasound/contrast-enhanced US: High grade lesion can be compared, between the technologies. iUS an be used for the localization for most of the lesion, but with better results with cystic/heterogeneous tumors. Contrast-enhanced US has good visualization in vascularized tumors and give intra-operative vascular aspects. Images from Prada et al. ${ }^{[51]}$

technology. ${ }^{[45-47]}$

Intra-operative ultrasound is more effective with heterogeneous lesions, with cystic compartments, and lesions with different echogenicity from the cortex, important in deep lesions, more than $1 \mathrm{~cm}$ from the cortex. Several reports support the use of iUS with good results such as good visualization of tumor borders with 3D iUS in $88 \%$ of patients and had led to an EOR in $55 \%,{ }^{[48]}$ numbers compared to the use of iMRI and 5-ALA. ${ }^{[49,50]}$

Therefore, the use of US contrast in brain tumor surgery, called intra-operative contrast-enhanced US (ICEUS), is to determine better tumor visualization and also vascularization, is in study. ${ }^{[51]}$ The contrast agents containing microbubbles hit by low-acoustic power US waves resonate with a specific value that can be read by a US algorithm for contrast. $^{[52,53]}$ There is a good correlation between the preoperative MRI and iUS and can reach a small difference of $2 \mathrm{~mm}$ with the advantage of being intra-operative and dynamic [Figure 4]. Nevertheless, neither iUS nor ICEUS can provide good borders for all LGG because of the similar echogenicity between the tumor and normal tissue. ${ }^{[1]}$ Differently from the contrast-MRI, the ICEUS depends on intra-vascular micro bubbles resonance, which will not provide the interstitial aspects. Fluorescence-guided surgery such as the use of 5-ALA can highlight tumor borders, but only in high-grade gliomas. Compared with 5-ALA and iMRI, the iUS has the advantage of providing borders images not only for high grade gliomas, but also for other types of tumor such as metastasis, meningeomas and some LGG, and the relationship to normal/vascular tissue with the non-stop surgery advantage. ${ }^{[51]}$

Fewer studies have shown the capability of the iUS and MRI; ${ }^{[48]}$ further studies are needed to evaluate the real aspect of the ICEUS and the use of combined methods with hybrid probe with MRI neuronavigation and iUS.

\section{Fluorescence guidance}

Even though intra-operative image guidance has evolved

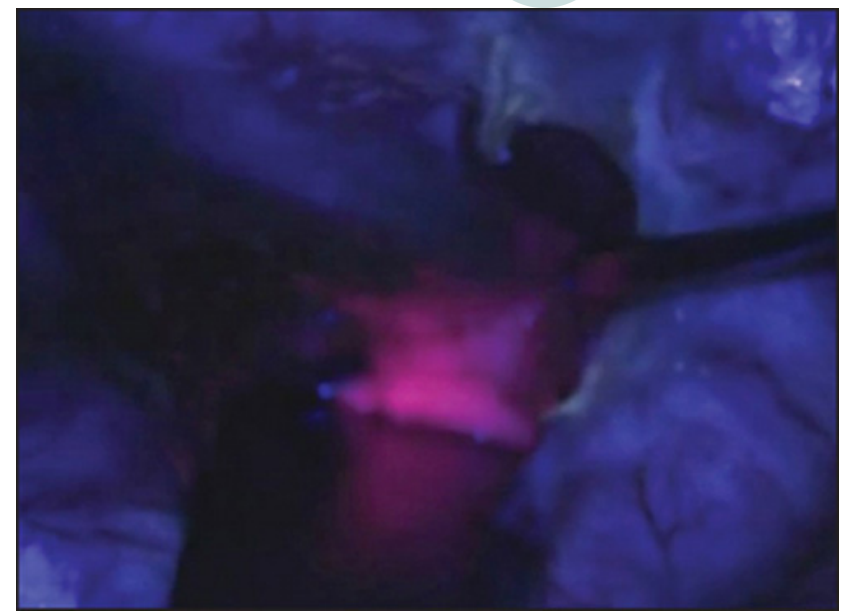

Figure 5: Aminolevulinic acid: the use of 5-aminolevulinic acid in high grade glioma. The tumor has the pink aspect and the normal brain in dark blue

the past decades, the interface between tumor borders, remaining tumor cells, and normal tissue is challenging.

Despite several substances have been studied, there are two major promising fluorescences: 5-ALA and sodium fluorescein.

The administration of 5-ALA leads to differential accumulation of protoporphyrin in the malignant tissues, via heme-biosyntheses pathway. ${ }^{[54,55]}$

The 5-ALA administration has proved to increase the GTR of glioblastomas ( $65 \%$ vs. $36 \%$; $\mathrm{P}<0.0001)$, smaller volume of the residual tumor (medians $0 \mathrm{~cm}^{3} v s .0 .7 \mathrm{~cm}^{3} ; P$ $<0.0001$ ), and better progression-free survival in 6 months after intervention (41\% vs. 21\%: $P=0.0003$ ) [Figure 5]. Recently, such beneficial results were corroborated by the assessment of 251 eligible cases from 18 clinics; they demonstrated greater proportions of complete resections of malignant gliomas with the use of 5-ALA (67\% vs. 45\%; $P=0.000)$ and progression-free survivors in 6 months after removal of glioblastoma ( $69 \%$ vs. $48 \% ; P=0.002)$, which corroborated with previous studies. ${ }^{[54,56,57]}$ Studies of fluorescence guidance combined with neuronavigation and brain mapping shows rates up to $98 \%$ of GTR in selected cases. ${ }^{[58,59]}$ In addition, the fluorescence guidance may reach beyond contrast-enhanced tumor borders and infiltrative zones that might be shown in the fluid attenuation inversion recovery (FLAIR) sequences of MRI. ${ }^{[42]}$ Although 5-ALA might be promising, it has some issues to be considered. First, we have to consider its high sensitivity and a lowspecificity, in special the non-high intensity pigmentations areas of fluorescein such as in tumor border and the hyperpigmentation in non-tumoral areas (necrosis, fibrosis, astrocytes infiltration) and also other non-glial lesios as lymphoma and metastasis. Furthermore, the absence of tissue fluorescence is common in LGG due to its relatively unruptured blood-brain-barrier and other intrinsic mechanisms of fast elimination of the drug; this makes it useless for LGG surgery. ${ }^{[1,60-63]}$ Moreover, the studies with 
fluorescence guidance have not studied the tumor genes and the good results could be genetically related. Further studies are needed to direct correlate the genetically aggressive tumors and the use of 5-ALA.

Another substance used for guidance is the sodium fluorescence, which accumulates in high neovascularization areas, also seen in high-grade lesions. Recent studies point to an increase of EOR and GTR, but without increasing of the overall survival rate. ${ }^{[64]}$ After review, 5-ALA had 91\% sensitivity, 59\% specificity, $85 \%$ PPV, and $71 \%$ negative predictive value for histopathological identification of malignant glioma. ${ }^{[65]}$ Future objectives in fluorescence guidance may lead to better microscopic visualization methods for the fluorescein such as filters, special masks or lens. ${ }^{[66]}$

\section{Current evidences}

The Cochrane group has reviewed all the reports of image-guided surgery for brain tumor resection and found some issues. Most of the studies are not controlled and randomized; also patients' baselines and tumor aspects were heterogeneous in most of the groups and the resecability of them was different between intervention and control groups. ${ }^{[2]}$ Despite limitations and low quality of evidence, the analyses from the classical reports from Senft 2011, Stummer 2006 and Wu 2007 showed a trend for better results. ${ }^{[2]}$ Complete tumor resection was achieved with iMRI in 23/24 (96\%) of participants in the intervention arm group compared with $17 / 25(68 \%)$ of participants in the control arm (relative risk [RR] for incomplete resection $0.13,95 \%$ confidence interval $[\mathrm{CI}]$ : $0.02-0.96$, low quality evidence). ${ }^{[2]}$

Using 5-ALA, complete resection was performed in 90/139 $(65 \%)$ of the intervention arm vs. 47/131 (36\%) of the control arm (RR for incomplete resection 0.55 , 95\% CI: $0.42-0.71$, low quality evidence). Finally, neuronavigation with DTI was achieved among the 85 participants with high-grade glioma and complete tumour resections were achieved in 32/42 in the DTI arm vs. 14/43 in the control arm (RR for incomplete resection 0.35, 95\% CI: 0.20-0.63, very low quality evidence). Among 129 participants with LGG, complete tumor resections were achieved in 40/61 in the DTI arm vs. 42/68 in the control arm (no significant difference). ${ }^{[2]}$ In survival analysis, the 5-ALA groups had a median survival of 15.2 months (95\% CI: 12.9-17.5) in intervention group and control with 13.5 months $(95 \%$ CI: 12.0-14.7). The neuronavigation-DTI arm was 21.2 months (95\% CI: 14.1-28.3) vs. 14.0 months (95\% CI: 10.217.8). Only in World Health Organization grade IV tumors analysis, neuronavigation-DTI arm was 19.3 months (95\% CI: 15.2-23.5) vs. 11.1 months (95\% CI: 7.3-15.2) in the control arm. ${ }^{[2]}$ In time to progression, the median time in iMRI group was 226 days (95\% CI: 0.0-454) vs. 154 days (95\% CI: 60-248) in control. With 5-ALA, it was 5.1 months (95\% CI: 3.4-6.0) vs. 3.6 months (3.2-4.4 months) in control. ${ }^{[2]}$ It is clear that the group analysis was not homogeneous and it might be due to a lack of protocols and a standardized approach to all lesions. Furthermore, there is need for standardization of reports for a systematic-review analysis and for future trends. Even though, the theorical benefits of the novel techniques should impulse more randomized, controlled trials with better baselines.

\section{Future technologies}

Neuronavigation has become more popular and the localization of tumors has come to practice with the navigation instrument and the monitor. Even though, what if we had the images seen in the surgical field continuously, without navigators? The augmented reality has come to time with the objective of sending information to surgical field without monitors.

Augmented reality technique has four steps: virtual image creation; real environment; projection and registration. Thus, image can be seen in the surgical field and the virtual interface can be used. The augmented reality is important in planning surgery and having the lesion visible in the skin since the beginning of the surgery. The augmented reality can be applied not only to the surgical field to prepare a better surgical incision and approach, but also to the surgical view in the microscope, which is important when the surgeon cannot take his or her eyes/instruments from the microscopic field. ${ }^{[67,68]}$

Moreover, the augmented reality could also include other parameters such as fiber tracts or important structures that should not be approached. As an innovation in neurosurgical surgery, there are few studies but promising applications. ${ }^{[67]}$

Also other interesting concept is the regional vs. global DTI biomarkers for glioblastoma. Most of this lesions are heterogeneous with multiple histological features and can lead to different degrees of malignancy, thus biopsies can be different in multiple areas. DTI is routinely used to locate high-grade areas, but the development of a sensitive and specific biomarker, remains an issue. Also, the role of DTIderived tensor metrics in normal brain and infiltrated brain is important for the distinction of tumor infiltration in noncontrast-enhanced areas. As the GBM been considered as a whole brain disease, DTI analysis of the whole brain might be more interesting than studying just the lesion areas. Roldán-Valadéz et al. showed that relative anisotropy, axial diffusivity (AD), $\mathrm{Cl}$ (linear tensor), Cs (spherical tensor), were important for regional DTI tumor analysis. ${ }^{[69]}$ Also, Cortez-Conradis pointed for $\mathrm{AD}, \mathrm{Cl}, \mathrm{Cs}$ and introduced the whole brain concept. The advantages of whole brain DTI analysis are: Decrease of bias associated with the analysis of just one region of interest; the tumor and edema regions are included; lesions not perceived by the radiologist's eye on conventional sequences would be included in a global assessment; it may avoid problems associated with partial volume effects, and inaccurate image coregistrations. ${ }^{[70]}$ 
Furthermore, these biomarkers could also been applied for other tumors and even other neurological diseases, without any contrast addition and increase of costs. ${ }^{[69,70]}$

For high-grade lesions with increased neo-vascularization, there was a report with use of indocyanine green (ICG) for detection of tumor borders. It is classically used by ophthalmologists for retinal vasculature and more recently for vascular neurosurgeries for aneurysms and arteriovenous malformations; however, for surgical borders for high-grade gliomas, it is a novel technique. ${ }^{[71]}$ Eyüpoglu et al. reported the ability of demonstrating the hypervascular areas with ICG that were not visible with the 5-ALA use. This technique was called dual intra-operative visualization approach (DIVA) with the initial approach using 5-ALA; after all initial tumor was resected, ICG was administered for visualization of remaining hypervascularization areas, with good initial results. Further studies are needed, but DIVA technique could be an interesting approach for further resection of non-fluorescein areas. ${ }^{[72]}$

One of the most difficult tasks in glioma surgery is the low-grade lesion. Most of the low-grades have similar density, echogenicity, and macroscopic aspect. Despite the neuronavigation progression, there are few MRI methods for low-grade tumor visualization, and most of the times the lesion is not contrast-enhanced and there is just the FLAIR sequence for tumor borders. ${ }^{[73]}$ Ramakrishna et al. showed improvement of overall survival with aggressive resection of FLAIR tumor limits, not only in the first attempt, but also in reoperation, regardless of patient age, pathology, chemotherapy, and radiation. ${ }^{[74]}$

The 5-ALA for LGGs is usually reported as non-visible, but it is not true for all of them. Valdés showed that $5 / 12$ patients had at least 1 instance of visible fluorescence during surgery and $45 \%$ of the non-visible fluorescence had a higher and detectable concentration of PpIX in the tumor tissue after the 5-ALA administration. With this idea, other researches were made to accurate the visibility of the fluorescein, or guide the elevated concentration in tissue with special probes of light visualization or high-resolution microscopic techniques, but with few results by this date. ${ }^{[75]}$

\section{CONCLUSION}

Evidences of the correlation between tumor removal and increase of survival rate have an impulse in novel technologies for safe resection and EOR. The uses of iMRI, DTI, PET, iUS, and fluorescence guidance have come to establish the neuronavigation era in neurosurgery.

Also, there is an increasing importance of the tumor genetics and behavior, which will provide crucial information and will guide tumor resection and adjuvant treatment. Despite all limitations of each technology and the lack of clear evidences, it is clear that this neurosurgeon/technology interface has come tighter and promising. However, the best result will come with the integration between technology for resection and tumor nature knowledge.

\section{Financial support and sponsorship} Nil.

\section{Conflicts of interest}

There are no conflicts of interest.

\section{REFERENCES}

1. D'amico RS, Kennedy BC, Bruce JN. Neurosurgical oncology: advances in operative technologies and adjuncts. $J$ Neurooncol 2014;119:451-63.

2. Barone DG, Lawrie TA, Hart MG. Image guided surgery for the resection of brain tumours. Cochrane Database Syst Rev 2014; doi: 10.1002/14651858.

3. Lacroix M, Abi-Said D, Fourney DR, Gokaslan ZL, Shi W, DeMonte F, Lang FF, McCutcheon IE, Hassenbusch SJ, Holland E, Hess K, Michael C, Miller D, Sawaya R.. A multivariate analysis of 416 patients with glioblastoma multiforme: prognosis, extent of resection, and survival. J Neurosurg 2001; 95:190-8.

4. Sanai N, Polley MY, McDermott MW, Parsa AT, Berger MS. An extent of resection threshold for newly diagnosed glioblastomas. $J$ Neurosurg 2011;115:3-8.

5. Sanai N, Berger MS. Glioma extent of resection and its impact on patient outcome. Neurosurgery 2008;62:753-64; discussion 264-6.

6. Vigneswaran K, Neill S, Hadjipanayis CG. Beyond the World Health Organization grading of infiltrating gliomas: advances in the molecular genetics of glioma classification. Ann Transl Med 2015;3:95.

7. Rudà R, Pellerino A, Magistrello M, Franchino F, Pinessi L, Soffietti R. Molecularly Based Management of gliomas in clinical practice. Neurol Sci 2015;36:1551-7.

8. Eckel-Passow JE, Lachance DH, Molinaro AM, Walsh KM, Decker PA, Sicotte H, Pekmezci M, Rice T, Kosel ML, Smirnov IV, Sarkar G, Caron AA, Kollmeyer TM,Praska CE, Chada AR, Halder C, Hansen HM, McCoy LS, Bracci PM, Marshall R, Zheng S, Reis GF, Pico AR, O’Neill BP, Buckner JC, Giannini C, Huse JT,Perry A, Tihan T, Berger MS, Chang SM, Prados MD, Wiemels J, Wiencke JK, Wrensch MR, Jenkins RB. Glioma Groups Based on 1p/19q, IDH and TERT Promoter Mutations in Tumors. N Engl Med 2015;372:2499-508.

9. Fontana EJ, Benzinger T, Cobbs C, Henson J, Fouke SJ. The evolving role of neurological imaging in neuro-oncology. $J$ Neurooncol 2014; 119:491-502.

10. Johnson RD, Stacey RJ. The impact of new imaging technologies in neurosurgery. Surgeon 2008; 6:344-9.

11. Grossman SA Ye X, Piantadosi S, Desideri S, Nabors LB, Rosenfeld M, Fisher J. Survival of patients with newly diagnosed glioblastoma treated with radiation and temozolomide in research studies in the United States. Clinical Cancer Res 2010;16:2443-9.

12. Stupp R, Tonn JC, Brada M, Pentheroudakis G. ESMO Guidelines Working Group.High-grade malignant glioma: ESMO Clinical Practice Guidelines for diagnosis, treatment and follow-up. Ann Oncol 2010;21 Suppl 5:v190-3.

13. Stupp R, Hegi M, Weller. Neuro-oncology, a decade of temozolomide and beyond. Expert Rev Anticancer Ther 2010;1675-7.

14. Dandy WE. Removal of right cerebral hemisphere for certain tumors with hemiplegia: Preliminary report. JAMA 1928; 90:823-5.

15. Dandy WE. Physiological studies following extirpation of the right cerebral hemisphere in man. Bull Johns Hopkins Hosp 1933;53:31-51.

16. Talacchi A, Santini B, Casagrande F, Alessandrini F, Zoccatelli G, 
Squintani GM. Awake surgery between art and science. Part I: clinical and operative settings. Funct Neurol 2013;28: 205-21.

17. Talacchi A, Santini B, Casartelli M, Monti A, Capasso R, Miceli G. Awake surgery between art and science. Part II: language and cognitive mapping . Funct Neurol 2013;28: 223-9.

18. Shinoura N, Midorikawa A, Yamada R, Hana T, Saito A, Hiromitsu K, Itoi C, Saito S, Yagi K. Awake craniotomy for brain lesions within and near the primary motor area: A retrospective analysis of factors associated with worsened paresis in 102 consecutive patients. Surg Neurol Int 2013;4:149.

19. Ojemann JG, Miller JW, Silbergeld DL. Preserved function in brain invaded by tumor. Neurosurgery 1996;39:253-8.

20. Bello L, Acerbi F, Giussani C, Baratta P, Taccone P, Songa V, Fava M, Stocchetti N, Papagno C, Gaini SM. Intraoperative language localization in multilingual patients with gliomas. Neurosurgery 2006; 59:115-25.

21. Lowestein PR, Castro MG. Pushing the limits of glioma resection using electrophysiologic brain mapping. J Clin Oncol 2012;30:2437-40.

22. Li T, Bai H, Wang G, Wang W, Lin J, Gao H, Wang L, Xia L, Xie X. Glioma localization and excision using direct electrical stimulation for language mapping during awake surgery. Exp Ther Med 2015;9:1962-6.

23. De Witt Hamer PC, Robles SG, Zwinderman AH, Duffau H, Berger MS. Impact of intraoperative stimulation brain mapping on glioma surgery outcome: a meta-analysis. J Clin Oncol 2012;30:2559-65.

24. De Benedictis A, Sarubbo S, Duffau H. Subcortical surgical anatomy of the lateral frontal region: Human white matter dissection and correlations with functional insights provided by intraoperative direct brain stimulation: laboratory investigation. $J$ Neurosurg 2012;117:1053-69.

25. Ilmberger J, Ruge M, Kreth FW, Briegel J, Reulen HJ, Tonn JC. Intraoperative mapping of language functions: A longitudinal neurolinguistic analysis. $J$ Neurosurg 2008;109:583-92.

26. Lüders HO. Symptomatic Areas and Electrical Cortical Stimulation. New York: Churchill Livingstone, 2000.

27. Duffau H. Brain mapping in tumors: Intraoperative or extraoperative? Epilepsia 2013;54:79-83.

28. Gras-Combe G, Moritz-Gasser S, Herbet G, Duffau H. Intraoperative subcortical electrical mapping of optic radiations in awake surgery for glioma involving visual pathways. J Neurosurg 2012;117:466-73.

29. Maldonado IL, Moritz-Gasser S, de Champfleur NM, Bertram L, Moulinié G, Duffau H. Surgery for gliomas involving the left inferior parietal lobule: new insights into the functional anatomy provided by stimulation mapping in awake patients. J Neurosurg 2011;115:770-9.

30. Ogawa S, Lee TM, Kay AR, Tank DW. Brain magnetic resonance imaging with contrast dependent on blood oxygenation. Proc Natl Acad Sci USA 1990; 87: 9868-72.

31. Moseley ME, Cohen Y, Kucharczyk J, Mintorovitch J, Asgari HS, Wendland MF, Tsuruda J, Norman D. Diffusion-weighted MR imaging of anisotropic water diffusion in cat central nervous system. Radiology 1990; 176: 439-46.

32. Jellison BJ, Field AS, Medow J, Lazar M, Salamat MS, Alexander AL. Diffusion tensor imaging of cerebral white matter: a pictoral review of physics, fiber tract anatomy, and tumor imaging patterns. AJNR Am J Neuroradiol 2004; 25: 356-69.

33. Wu JS, Zhou LF, Tang WJ, Mao Y, Hu J, Song YY, Hong XN, Du GH. Clinical evaluation and follow-up outcome of diffusion tensor imaging-based functional neuronavigation: a prospective, controlled study in patients with gliomas involving pyramidal tracts. Neurosurgery 2007;61:935-48; discussion 948-9.

34. Kuhnt D, Bauer MH, Nimsky C. Brain shift compensation and neurosurgical image fusion using intraoperative MRI: current status and future challenges. Crit Rev Biomed Eng 2012;40:175-85.

35. Kremer P, Tronnier V, Steiner HH, Metzner R, Ebinger F, Rating D, Hartmann M, Seitz A, Unterberg A, Wirtz CR. Intraoperative MRI for interventional neurosurgical procedures and tumor resection control in children. Childs Nerv Syst 2006; 22:674-8.

36. Senft C, Franz K, Ulrich CT, Bink A, Szelényi A, Gasser T, Seifert V. Low field intraoperative MRI-guided surgery of gliomas: a single center experience. Clin Neurol Neurosurg 2010;112:237-43.

37. Fahlbusch R, Ganslandt O, Buchfelder M, Schott W, Nimsky C. Intraoperative magnetic resonance imaging during transsphenoidal surgery. J Neurosurg 2001;95:381-90.

38. Kubben PL, ter Meulen KJ, Schijns OE, ter Laak-Poort MP, van Overbeeke JJ, van Santbrink H. Intraoperative MRI-guided resection of glioblastoma multiforme: a systematic review. Lancet Oncol 2011;12:1062-70.

39. Senft C, Seifert V, Hermann E, Franz K, Gasser T. Usefulness of intraoperative ultra low-field magnetic resonance imaging in glioma surgery. Neurosurgery 2008;63:257-66; discussion 266-7.

40. Foroglou N, Zamani A, Black P. Intra-operative MRI (iop-MR) for brain tumour surgery. Br J Neurosurg 2009;23:14-22.

41. Gerlach R, du Mesnil de Rochemont R, Gasser T, Marquardt G, Reusch J, Imoehl L, Seifert V. Feasibility of Polestar N20, an ultralow- field intraoperative magnetic resonance imaging system in resection control of pituitary macroadenomas: lessons learned from the first 40 cases. Neurosurgery 2008;63:272-84; discussion 284-5.

42. Roder C, Bisdas S, Ebner FH, Honegger J, Naegele T, Ernemann $\mathrm{U}$, Tatagiba M. Maximizing the extent of resection and survival benefit of patients in glioblastoma surgery: High-field iMRI versus conventional and 5-ALA-assisted surgery. Eur J Surg Oncol 2014;40:297-304.

43. Knauth M, Aras N, Wirtz CR, Dörfler A, Engelhorn T, Sartor K. Surgically induced intracranial contrast enhancement: potential source of diagnostic error in intraoperative MR imaging. AJNR Am J Neuroradiol 1999;20:1547-53.

44. Özduman K, Yıldız E, Dinçer A, Sav A, Pamir MN. Using intraoperative dynamic contrast-enhanced T1-weighted MRI to identify residual tumor in glioblastoma surgery. $J$ Neurosurg 2014;120-:60-6.

45. Rygh OM, Selbekk T, Torp SH, Lydersen S, Hernes TA, Unsgaard G. Comparison of navigated 3D ultrasound findings with histopathology in subsequent phases of glioblastoma resection. Acta Neurochir (Wien) 2008;150:1033-41; discussion 1042.

46. Selbekk T, Jakola AS, Solheim O, Johansen TF, Lindseth F, Reinertsen I, Unsgård G. Ultrasound imaging in neurosurgery: approaches to minimize surgically induced image artefacts for improved resection control. Acta Neurochir (Wien) 2013;155:973-80.

47. Coenen VA, Krings T, Weidemann J, Hans FJ, Reinacher P, Gilsbach $\mathrm{JM}$, Rohde V. Sequential visualization of brain and fiber tract deformation during intracranial surgery with three dimensional ultrasound: an approach to evaluate the effect of brain shift. Neurosurgery 2005;56:133-41; discussion 133-41.

48. Moiyadi AV, Shetty PM, Mahajan A, Udare A, Sridhar E. Usefulness of three-dimensional navigable intraoperative ultrasound in resection of brain tumors with a special emphasis on malignant gliomas. Acta Neurochir 2013;155-: 2217-25.

49. Senft C, Bink A, Franz K, Vatter H, Gasser T, Seifert V. Intraoperative MRI guidance and extent of resection in glioma surgery: a randomised, controlled trial. Lancet Oncol 2011;12:997-1003.

50. Díez Valle R, Tejada Solis S, Idoate Gastearena MA, García de Eulate R, Domínguez Echávarri P, Aristu Mendiroz J. Surgery guided by 5-aminolevulinic fluorescence in glioblastoma: volumetric analysis of extent of resection in single-center experience. $J$ Neurooncol 2011;102:105-13.

51. Prada F, Perin A, Martegani A, Aiani L, Solbiati L, Lamperti M, Casali C, Legnani F, Mattei L, Saladino A, Saini M, DiMeco F. Intraoperative Contrast-Enhanced Ultrasound for Brain Tumor Surgery. Neurosurgery 2014;74:542-52.

52. Quaia E. Assessment of tissue perfusion by contrast-enhanced 
ultrasound. Eur Radiol 2011;21:604-15.

53. Sidhu PS, Choi BI, Nielsen MB. The EFSUMB guidelines on the non-hepatic clinical applications of contrast enhanced ultrasound (CEUS): a new dawn for the escalating use of this ubiquitous technique. Ultraschall Med 2012;33:5-7.

54. Stummer W, Pichlmeier U, Meinel T, Wiestler OD, Zanella F, Reulen HJ. Fluorescence-guided surgery with 5-aminolevulinic acid for resection of malignant glioma: a randomised controlled multicentre phase III trial. Lancet Oncol 2006;7:392-401.

55. Valdés PA, Leblond F, Kim A, Harris BT, Wilson BC, Fan X, Tosteson TD, Hartov A, Ji S, Erkmen K, Simmons NE, Paulsen KD, Roberts DW. Quantitative fluorescence in intracranial tumor: implications for ALA-induced PpIX as an intraoperative biomarker. J Neurosurg 2011;115:11-7.

56. Tsugu A, Ishizaka H, Mizokami Y, Osada T, Baba T, Yoshiyama M, Nishiyama J, Matsumae M. Impact of combination of 5-Aminolevulinic Acid-induced Fluorescence with Intraoperative Magnetic Resonance Imaging-guided Surgery gor Glioma. World Neurosurg 2011;76:120-7.

57. Yamada S, Muragaki Y, Maruyama T, Komori T, Okada Y. Role of neurochemical navigation with 5-aminolevulinic acid during intraoperative MRI-guided resection of intracranial malignant gliomas. Clin Neurol Neurosurg 2015;130:134-9.

58. Della Puppa A, De Pellegrin S, d'Avella E, Gioffrè G, Rossetto M, Gerardi A, Lombardi G, Manara R, Munari M, Saladini M, Scienza R. 5-aminolevulinic acid (5-ALA) fluorescence guided surgery of high-grade gliomas in eloquent areas assisted by functional mapping. Our experience and review of the literature. Acta Neurochir (Wien) 2013; 155:965-72; discussion 972.

59. Schucht P, Beck J, Abu-Isa J, Andereggen L, Murek M, Seidel K, Stieglitz L, Raabe A. Gross total resection rates in contem- porary glioblastoma surgery: results of an institutional protocol combining 5 -aminolevulinic acid intraoperative fluorescence imaging and brain mapping. Neurosurgery 2012;71:927-35; discussion 935-6.

60. Li Y, Rey-Dios R, Roberts DW, Valdés PA, Cohen-Gadol AA. Intraoperative fluorescence-guided resection of high-grade gliomas: a comparison of the present techniques and evolution of future strategies. World Neurosurgery 2014;82:175-85.

61. Roberts DW, Valdés PA, Harris BT, Fontaine KM, Hartov A, Fan X, Ji S, Lollis SS, Pogue BW, Leblond F, Tosteson TD, Wilson BC, Paulsen KD. Coregistered fluorescence-enhanced tumor resection of malignant glioma: relationships between delta-aminolevulinic acid-induced protoporphyrin IX fluorescence, magnetic resonance imaging enhancement, and neuropathological parameters. Clinical article. J Neurosurg 2011; 114:595-603.

62. Miyatake S, Kuroiwa T, Kajimoto Y, Miyashita M, Tanaka H, Tsuji M. Fluorescence of non-neoplastic, magnetic resonance imagingenhancing tissue by 5 -aminolevulinic acid: case report. Neurosurgery 2007;61:E1101-3; discussion E1103-4.

63. Grossman R, Nossek E, Shimony N, Raz M, Ram Z. Intraoperative 5 -aminolevulinic acid- induced fluorescence in primary central nervous system lymphoma. J Neurosurg 2014;120:67-9.

64. Koc K, Anik I, Cabuk B, Ceylan S. Fluorescein sodium-guided surgery in glio- blastoma multiforme: a prospective evaluation. $\mathrm{Br} J$ Neurosurg 2008;22:99-103.

65. Zhao S, Wu J, Wang C, Liu H, Dong X, Shi C, Shi C, Liu Y, Teng L, Han D, Chen X, Yang G, Wang L, Shen C, Li H. Intraoperative Fluorescence-Guided Resection of High-Grade Malignant Gliomas Using 5- Aminolevulinic Acid-Induced Porphyrins: A Systematic Review and Meta-Analysis of Prospective Studies. PLOS ONE 2013;8:e63682.

66. Meza D, Wang D, Wang Y, Borwege S, Sanai N, Liu JT. Comparing high-resolution microscopy techniques for potential intraoperative use in guiding low-grade glioma resections. Lasers Surg Med 2015;47289-95.

67. Tabrizi LB, Mahvash M. Augmented reality-guided neurosurgery: accuracy and intraoperative application of an image projection technique. J Neurosurg 2015;123:206-11.

68. Mahvash M, Besharati Tabrizi L: A novel augmented reality system of image projection for image-guided neurosurgery. Acta Neurochir (Wien) 2015;155:943-7.

69. Roldán-Valadéz E, Ríos C, Cortez-Conradis D, Favila R, MorenoJimenez S. Global diffusion tensor imaging derived metrics differentiate glioblastoma multiforme $v s$. normal brains by using discriminant analysis: introduction of a novel whole-brain approach. Radiol Oncol 2014;48:127-36.

70. Cortez-Conradis D, Favila R, Isaac-Olive K, Martínez-López M, Ríos C, Roldán-Valadéz E. Diagnostic performance of regional DTIderived tensor metrics in glioblastoma multiforme: simultaneous evaluation of p, q, L, Cl, Cp, Cs, RA, RD, AD, mean diffusivity and fractional anisotropy. Eur Radiol 2013;23:1112-21.

71. Wang M, Serak J, Burks SS. Dual Intraoperative Visualization Approach Surgery: A Novel Technique Enhances Intraoperative Glioma Visualization. Neurosurgery 2015;77:24-5.

72. Eyüpoglu IY, Hore N, Fan Z, Buslei R, Merkel A, Buchfelder M, Savaskan NE. Intraoperative vascular DIVA surgery reveals angiogenic hotspots in tumor zones of malignant gliomas. Sci Rep 2015;5:7958.

73. Hollon T, Hervey-Jumper SL, Sagher O, Orringer DA. Advances in the surgical management of Low-Grade Glioma. Semin Radiat Oncol 2015;25:181-8.

74. Ramakrishna R, Hebb A, Barber J, Rostomily R, Silbergeld D. Outcomes in Reoperated Low-Grade Gliomas. Neurosurgery 2015;77:175-84; discussion 184

75. Valdés PA, Jacobs V, Harris BT, Wilson BC, Leblond F, Paulsen KD, Roberts DW. Quantitative fluorescence using 5-aminolevulinic acidinduced protoporphyrin IX biomarker as a surgical adjunct in lowgrade glioma surgery. J Neurosurg 2015;123:771-80. 